\title{
Relación entre los Factores Motivacionales, la Edad y el Sexo en las Personas Participantes de un Proyecto de Natación
}

\author{
Relation between Motivational Factors, Age, and Gender of Individuals Participating \\ in a Swimming Project
}

\author{
Alejandro Rodríguez Montero, María Morera, Kristy Barrantes Brais y \\ José Alexis Ugalde Ramírez. \\ Escuela Ciencias del Movimiento Humano y Calidad de Vida, Universidad \\ Nacional, Costa Rica \\ alrodri@una.cr
}

\begin{abstract}
Resumen
El propósito de esta investigación fue determinar la relación entre factores motivacionales para la práctica de la natación, según sexo y edad en las personas participantes en un proyecto de natación. En el estudio participaron 107 sujetos $(71 \%)$ del total de la población activa del proyecto, de los cuales 51 fueron

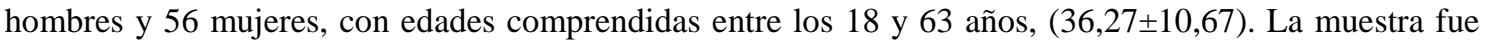
distribuida en cuatro categorías de edad (18 a 29 años; 30 a 39 años; 40 a 49 años y 50 años en adelante) y por sexo. Para conocer los motivos que inducen a las personas a realizar ejercicio físico, se aplicó el cuestionario Autoinforme de Motivos para la Práctica de Ejercicios Físicos (AMPEF), adaptado por Capdevilla Niñerola y Pintanel (2004). Los resultados del análisis individual demostraron que los factores: salud y prevención positiva; bienestar y diversión; fuerza y resistencia muscular, son motivos importantes tanto en hombres como mujeres en todas las categorías por edad, con valores $\geq 7$ (en una escala Likert de 1 a 10). No se encontró diferencias significativas en el puntaje total entre variables. Los datos registrados son consistentes con lo reportado en la literatura científica. Se concluye que los principales motivos de las personas para realizar actividad física son: salud, diversión, bienestar y mejoría de aspectos físicos, asimismo se encontró que existen diferencias significativas en los motivos relacionados con el desafío y la competencia, entre hombres y mujeres, resultados que también con coinciden con publicaciones previas.
\end{abstract}

Palabras Claves: motivación, salud, natación, actividad física

\begin{abstract}
The purpose of this study was to determine the relationship between motivational factors, sex and age on the participants at a project of swimming. The study included 107 subjects $(71 \%)$ of the total of active participants in the project, of which 51 were men and 56 women, aged between 18 and 63 years $(36,27 \pm$ 10,67). The sample was divided into four age categories (18-29 years, 30-39 years, 40-49 years and 50 and over) and by sex. To know the reasons that lead people to engage physical exercise, self-report questionnaire Motives for the Practice of Physical Exercises (AMPEF), adapted by and Pintanel Niñerola Capdevila (2004) was applied. The results of the individual analysis showed that the factors: positive health and prevention; well-being and fun; muscular strength and endurance are important reasons for both men and women in all age categories, with values $\geq 7$ (between 1 to 10). There were no significant differences in the total score between variables. The data reported are consistent with those reported in the literature. It is concluded that the main reasons for people to be physically active are: health, fun, wellbeing and improvement of physical, also found significant differences in the reasons relating to the challenge and competition between men and women, results also agree with previous publications.
\end{abstract}

Key Words: motivation, health, swimming, physical activity

Revista MHSalud® (ISSN: 1659-097X) Vol. 11. No. 1. Setiembre-Enero, 2014 Artículo de la Revista MHSalud de la Universidad Nacional, Costa Rica protegido por Licencia de Costa Rica 


\section{Introducción}

La motivación humana está relacionada con el desarrollo y funcionamiento de la personalidad dentro de los contextos sociales en los que se desenvuelve una persona (Deci y Ryan, 2000). De esta manera, se puede definir la motivación como el proceso por el cual el sujeto se plantea un objetivo, utiliza los recursos adecuados y mantiene una determinada conducta, con el propósito de lograr una meta (Benzi y Polani, 2000; Weiss y Amorose, 2008). El término motivación se entiende también como la fuerza que influye en un organismo para que inicie y dirija la conducta (Petri y Govern, 2006).

Partiendo de este punto, los motivos de participación hacia el ejercicio físico se encuentran asociados con la regulación de la conducta, y a la vez, son influenciados por la personalidad de cada individuo, por variables sociales y ambientales, las cuales inciden la elección de una u otra actividad (Cervelló, Escartí y Guzmán, 2007; Ingledew y Markland, 2008).

La motivación dentro del ámbito de la práctica de la actividad física y ejercicio físico, juega un papel determinante para la consecución de resultados y para mejorar tanto aspectos físicos como psicológicos (De Andrade, Salguero y Márquez, 2006; Salguero, GonzálezBoto, Tuero y Márquez, 2003). Asimismo los niveles elevados de motivación de las personas participantes en programas de actividad física, se relacionan con la adherencia de estas a dichos programas, factor a tomar en cuenta a la hora de obtener los beneficios a nivel físico y a nivel emocional a lo largo del tiempo (Blázquez y Feu, 2012; Moreno, Cervelló y González, 2007). Diversos autores (Granero, Gómez, Abraldes y Rodríguez, 2011; Moreno-Murcia, Borges, Marcos, Sierra y Huéscar, 2012; Pinto, Santos y Legani, 2012; Ruiz, García y Díaz, 2007), señalan que los principales motivos de las personas practicantes en programas de actividad física se relacionan con el ambiente social, diversión, salud, competencia, apariencia física y afiliación social.

Los motivos para que las personas se involucren en programas de actividad física varían según la edad (Moreno, Cervelló y Martínez, 2007; Moreno, Martínez, González-Cutre y Marcos, 2009; Pavón, Moreno, Gutiérrez y Sicilia 2004) y el sexo (Pavón y Moreno, 2008; Rodríguez-Romo, Boned y Garrido, 2009; Ruíz, García y Díaz, 2007). A mayor edad de las personas, predominan los motivos relacionados con el mantenimiento de la salud y la diversión (Gavala 2010; Pinto, Santos y Legnani, 2012; Rodríguez-Romo et al., 2009), así como la necesidad de la afiliación con otras personas (Moreno et al. 2007). Hombres y mujeres se comportan de manera distinta en cuanto a motivos para realizar actividad física (Pavón y Moreno, 2008; Rodríguez, Boned y Garrido, 2009; Ruíz, García y Díaz, 2007), los motivos de las mujeres se relacionan con el cuidado de la imagen y el control del peso corporal (Pavón y Moreno, 2008; Pinto et al., 2012). Los hombres presentan entre sus principales motivos la competición, el triunfo y el rendimiento físico (Águila, Sicilia, Muyor y Orta, 2009; Pavón y Moreno, 2008; Pinto et al., 2012).

Investigaciones señalan que para la práctica de natación, los motivos más frecuentes en la población adulta son: los beneficios para salud, la diversión, mantenerse en forma, prevenir enfermedades, la necesidad de desarrollar nuevas habilidades motoras, la necesidad de socialización (Andrade, Salguero, Márquez, 2006; Bíró, Füegedi y Revés, 2007; Hritz y 
Ramos, 2008; Moreno y Marín, 2002). Por lo que, para el favorecimiento de la adherencia al Proyecto de natación de la Escuela de Ciencias del Movimiento Humano y Calidad de Vida, Universidad Nacional es importante conocer los motivos que están conduciendo a las personas a practicar este tipo de ejercicio físico. Es por tanto de nuestro interés determinar la relación entre factores motivacionales, sexo y edad en las personas participantes en un proyecto de natación. Esto con el fin de plantear estrategias de mejoramiento, reforzamiento e inclusión de actividades dentro del agua que incremente la adherencia a la actividad física y ende se contribuya en una mejor calidad de vida y bienestar de nuestros clientes.

\section{Metodología}

\section{Participantes.}

La muestra fue seleccionada por conveniencia. Del total de 150 personas usuarias del Proyecto Natación para la Promoción de la Salud (NAPROSA), 107 (71\%) personas participaron en esta investigación, 51 fueron hombres y 56 mujeres, con edades comprendidas entre los 18 y 63 años, $(36,27 \pm 10,67)$. La muestra fue distribuida en cuatro categorías de edad (18 a 29; 30 a 39; 40 a 49 y 50 en adelante) y dividida por sexo. En la Tabla 1, se muestra los datos descriptivos según la edad y sexo. Las personas usuarias del Proyecto de Natación realizan semanalmente sesiones de diferentes progresiones y ejercicios de natación, con un tiempo aproximado de 55 minutos. Las sesiones son estructuradas de la manera tradicional, con una fase de calentamiento, una de trabajo principal y una de relajación.

Tabla 1

Promedios y desviaciones estándar de los hombres y mujeres participantes de acuerdo a la edad.

\begin{tabular}{lcccccc}
\hline \multirow{2}{*}{ Distribución por edades } & \multicolumn{3}{c}{ Mujeres } & \multicolumn{3}{c}{ Hombres } \\
\cline { 2 - 7 } & $n$ & Promedio & Desv. Típ. & $n$ & Promedio & Desv. Típ. \\
\hline 18 a 29 años & 15 & 25.3 & \pm 3.5 & 18 & 25 & \pm 3.04 \\
30 a 39 años & 17 & 34 & \pm 2.8 & 20 & 33.7 & \pm 2.3 \\
40 a 49 años & 14 & 44.1 & \pm 3.05 & 8 & 43.7 & $\pm 2,1$ \\
50 años en adelante & 10 & 54.4 & \pm 4.6 & 5 & 55.4 & \pm 3.4 \\
\hline
\end{tabular}

\section{Instrumentos.}

Los motivos fueron identificados por medio del cuestionario Autoinforme de Motivos para la Práctica de Ejercicios Físicos (AMPEF), adaptado por Capdevilla, Niñerola y Pintanel (2004). Este consta de 48 ítems, agrupados en 11 factores: peso e imagen corporal; diversión y bienestar; prevención y salud positiva; competición; afiliación; fuerza y resistencia muscular; reconocimiento social; control del estrés; agilidad y flexibilidad; desafío; y urgencias de salud. El formato de respuesta es tipo Likert de 0 a 10, siendo 0 nada verdadero y 10 totalmente verdadero. Según lo establece Niñerola, Capdevila y Pintanel (2006), cualquier factor es considerado determinante para realizar ejercicio físico cuando el promedio de dicho factor es $\geq 7$. 
Se reporta una validez y estabilidad en su estructura de 11 factores los cuales proveen un $72 \%$ de variabilidad del total de datos. Asimismo, la consistencia interna de la escala coeficientes 0,922 (alfa de Cronbach). La fiabilidad del instrumento, analizada mediante la estabilidad temporal a partir de la correlación de Pearson es de $(p<.001)$, siendo aceptables y significativas entre el test y el retest. Además, la correlación entre las puntuaciones totales mostradas es de 0,8 .

\section{Procedimiento.}

Primeramente se solicitó permiso a las autoridades del proyecto, las cuales demostraron su anuencia a participar de esta investigación. Seguidamente y conforme fueron llegando las personas usuarias a realizar su ejercicio físico, se les explicó los objetivos y alcances de la investigación y se les entregó el consentimiento informado. Únicamente las personas que firmaron el consentimiento pasaron a la siguiente fase, la cual fue la aplicación del instrumento AMPEF. De manera individual y en presencia del investigador, se le solicitó a cada persona que completara el cuestionario minutos antes de que realizara la sesión de natación. Esta aplicación se llevó a cabo en la piscina del lugar, en el cual se acondicionó un espacio con mesas y sillas para que pudieran contestarlo de manera cómoda. Una vez finalizado el cuestionario cada persona procedió a realizar su sesión práctica.

\section{Análisis estadístico.}

Se interpretaron los datos según lo recomendado por Niñerola et al, (2006), de manera individual (se utiliza para analizar el peso de cada ítem dentro del factor), factorial y total (se recomienda únicamente para estudios longitudinales). Para la interpretación individual, se analizó cada ítem de acuerdo a la puntuación resultante de la sumatoria respectiva de cada participante, y dividiéndola entre la cantidad de cuestionarios completados, obteniéndose un promedio general, un promedio para mujeres y otro para hombres, con sus respectivas desviaciones estándar. Este análisis descriptivo se llevó a cabo para cada factor, por medio de la hoja de cálculo Excel Microsoft Office 2007.

Las puntuaciones factoriales, se obtuvieron de la sumatoria de los ítems correspondientes a cada factor, dividida entre la cantidad de ítems que lo conformaba, obteniéndose un promedio general por factor. Para establecer la relación entre los factores motivacionales, se realizó un ANOVA factorial 2 (Sexo) x 4 (Edad) para cada factor y el valor total, en el programa estadístico SPSS versión 18. Además se realizó un análisis Post Hoc de efectos simples en caso de interacción significativa y para el caso de efectos simples significativos (una vía) se aplicó el Post Hoc de comparaciones múltiples de Bonferroni o el de mínimas diferencias significativas cuanto esto fuese necesario. Se estableció a priori el grado de significancia $p \leq 0.05$.

\section{Resultados}

En este estudio descriptivo y transversal se exploraron los factores motivacionales que prevalecen en esta población para la práctica de la natación, como medio para promover y mantener su salud. Se analizaron los resultados tomando en cuenta la edad y el sexo en interacción con los factores motivacionales. 
De manera descriptiva y según lo establece Niñerola, Capdevila y Pintanel (2006), cualquier factor cuyo valor es $\geq 7$ en el AMPEF es considerado determinante para la realización del ejercicio físico. Como se puede observar en la Tabla 2, los factores diversión y bienestar, prevención y salud positiva, control del estrés y fuerza y resistencia muscular son motivos importantes para la práctica deportiva en mujeres de 18 a 39 años de edad. En el caso de las mujeres de 30 años en adelante el factor peso e imagen corporal se convierten en un motivo importante para realizar actividad física. El factor agilidad y flexibilidad es relevante para mujeres de 40 en adelante. En particular, el factor desafío es esencial para mujeres de 40 a 49 años y el factor urgencias de salud para mujeres de 50 años en adelante. Todos los anteriores factores cuentan con un valor $\geq 7$.

En contra parte, los hombres en todos los grupos de edad presentan niveles de motivación asociados a los factores de prevención y salud positiva, diversión y bienestar, fuerza y resistencia muscular, agilidad y flexibilidad (ver Tabla 2). El factor peso e imagen corporal toma relevancia para hombres de 20 a 39 años. En el caso del factor control del estrés únicamente para el grupo de 40 a 49 años no es tan importante para el resto de las edades si es un factor para la práctica de actividad física. El factor afiliación es importante para los hombres de 50 años en adelante. Al mismo tiempo el factor desafío es considerado por los hombres de 20 a 29 años y los de 50 en adelante como un aspecto influyente en su práctica (ver Tabla 2 , valores $\geq 7$ ).

Tabla 2

Promedios y desviación estándar de cada factor según el sexo y las categorías de edad

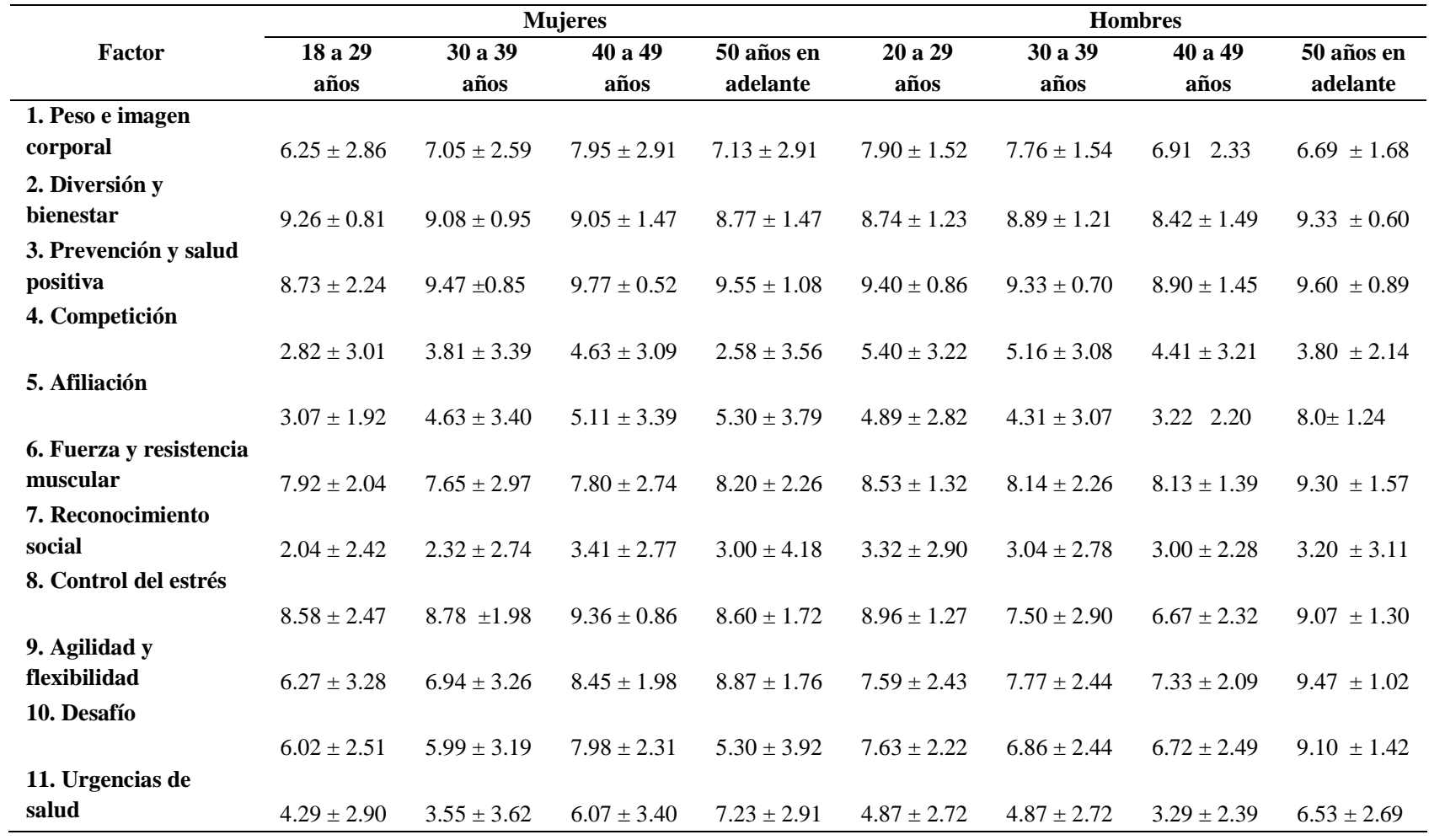

Seguidamente, se realizaron 12 ANOVAs factorial 2 (sexo) x 4 (edad). Los resultados mostraron una interacción en el factor control del estrés, $F(7,99)=12,3 p=0.037$. De acuerdo con al análisis Post Hoc aplicado se encontró efecto simple significativo de sexo 

únicamente en el grupo de edad de 40 a 49 años, siendo las mujeres quienes presentaron un puntaje promedio mayor que los hombres. Asimismo se encontró efecto simpe significativo de la edad solamente para el grupo de los hombres. Debido a esto se requirió un análisis Post Hoc de una vía (mínimas diferencias significativas) y se encontró que el grupo 18 a 29 años tuvo un promedio más alto que el grupo 30 a 39 años y que el grupo de 40 a 49 años. No se encontró otras diferencias significativas.

Asimismo, se encontró un efecto estadísticamente significativo entre sexo, en el factor desafío, $F(7,99)=4,85, p=0.03$, siendo las mujeres $(6,37 \pm 3,04)$ las que presentan menor desafío que los hombres $(7,32 \pm 2,33)$. Al mismo tiempo, se encontraron efectos estadísticamente significativos entre al menos dos de los grupos de edad, en el factor Urgencias de salud, $F(7,99)=2,71, p=0.049$, así como en el factor Afiliación, $F(7,99)=$ $2,76, p=0.046$. Posteriormente se realizó la prueba de seguimiento (post hoc) para comparaciones múltiples de Bonferroni. Los resultados demuestran que la diferencia significativa para el factor Urgencias de Salud, es entre la categoría de edad de 50 años en adelante $(6,88 \pm 2,76)$ y la categoría de 30 a 39 años de edad, (4,68 $\pm 3,30)$. En el factor Afiliación se encuentra entre la categoría de edad de 18 a 29 años $(3,98 \pm 2,58)$ y la categoría de edad de 50 años en adelante $(6,65 \pm 3,37)$.

\section{Discusión}

De manera general, y a partir del análisis descriptivo en esta muestra se identifica que la motivación para involucrarse en la natación se deriva de factores asociados con salud y bienestar desde perspectivas como la prevención, la diversión, el control del estrés y el mejoramiento de cualidades físicas (fuerza, resistencia, agilidad y flexibilidad). Esta tendencia coincide con lo encontrado en estudios previos que analizaron motivaciones relacionadas con actividades en el medio acuático (De Andrade et al., 2006; Moreno y Marín, 2002; Moreno, Martínez y Marcos, 2008). De igual manera los resultados obtenidos se asemejan con los reportados en otros estudios realizados en diferentes contextos de actividad física y deporte no competitivo en función al sexo y edad de los y las participantes (Granero et al., 2011; Jiménez-Torres, Goרdoy-Izquierdo y Godoy, 2012; Moreno et al., 2012; Pavón y Moreno, 2008). Por su parte, la natación se identifica como una de las actividades físicas a partir de la cual se pueden desarrollar procesos de promoción de la salud (Pérez y Devís, 2003), en los cuales se potencien factores asociados con el bienestar de las poblaciones (Goñi y Infante, 2010; Romero, Brustad y García, 2007).

Los factores motivacionales predominantes en esta investigación, se asocian con elementos de motivación intrínseca y de identificación (Deci y Ryan, 2000; Ingledew, Markland y Ferguson, 2009), lo cual difiere de lo encontrado en investigaciones previas, en las cuales la práctica de natación tenía como motivaciones principales aspectos de tipo extrínseco como hacer amigos, pertenecer a un equipo y disfrutar de actividades sociales (Guillén y Álvarez-Malé, 2010; Jepson et al., 2012). Partiendo de este punto, las personas que realizan actividades físicas en el medio acuático disfrutan de la realización de la misma, interviniendo para ellos factores que incrementan el bienestar y disfrute de las actividades acuáticas, así como, si el participante interviene en la toma de decisiones, elección y determinación de la actividad que desea realizar, se incrementaría en ellos la motivación intrínseca, por lo que la persona disfrutará más durante la realización de la actividad (Cid y Louro, 2010; Moreno et al., 2010). La natación como una forma de acerarse al mejoramiento 
de la imagen corporal pareciera ser relevante en mujeres adultas (Moreno et al., 2008; Moreno et al., 2009).

El factor control del estrés resultó más relevante para hombres entre 18 y 29 años y para mujeres entre 40 y 49 años. Las demandas psicosociales y los cambios fisiológicos podrían asociarse con estos hallazgos. Para los adultos jóvenes, las exigencias de cumplir con diversos roles pueden resultar en niveles de estrés que requieran de estrategias como la actividad física (Ingledew et al., 2009; Jiménez-Torres et al., 2012). Asimismo, el manejo del estrés es un aspecto relevante para el género femenino en general (Jeffrey, 2013) y en las etapas cercanas a la menopausia (Triviño, Stiepovich y Merino, 2007). Las actividades físicas están relacionadas con aspectos sociales, cuyos factores inciden en el estrés y tensión, por lo que se ha demostrado, que la práctica de ejercicio físico disminuye los niveles de estrés, lo que incentiva a que cada vez más personas deseen llevar una vida activa y saludable (Ingledew et al., 2009; Jiménez et al., 2008).

Los hombres en esta muestra reportaron mayor motivación asociada con factores de reto o desafío, en comparación con las mujeres. Previamente se ha documentado que la práctica de ejercicio o actividad física que demande algún tipo de reto, resulta ser atractiva para los hombres (Pavón y Moreno, 2008; Moreno et al., 2006; Pavón et al., 2004; Remor, 2007). La orientación a metas (Guillén y Álvarez-Malé, 2010) y la orientación al ego (Jeffrey, 2013) adquieren relevancia y pueden influir en la toma de decisiones con respecto a la participación en actividades físicas recreativas o competitivas (Medic, Young y Grove, 2013). De ahí, que este aspecto se encuentre perenne de alguna manera con mayor frecuencia en los varones al momento de ejercitarse, aunque, esto no indica que todo tipo de actividad física represente algún desafío para los hombres, ni tampoco que las mujeres no consideren desafiantes las actividades físicas que deciden realizar, asimismo, las actividades físicas en general se relacionan con características que permiten mostrar un crecimiento gradual en los niveles de involucración y desafío que representan las mismas para las personas practicantes (Romero, Brustad y García, 2007).

Para los participantes mayores de 50 años, lo cual incluye a las personas adultas mayores, los factores de afiliación (relacionados con el contacto y apoyo social) son significativamente más importantes que para los adultos jóvenes. El apoyo social y el fortalecimiento de las relaciones interpersonales constituyen aspectos fundamentales para la población adulta mayor (Duran, Orbegoz, Uribe-Rodríguez y Uribe, 2008) y se asocian con percepciones incrementadas de bienestar subjetivo (Mella et al., 2004) e indicadores de salud mental y física (González, Landero y Ruiz, 2008). En la población adulta joven parecieran tener más relevancia factores de tipo intrínseco al realizar actividad física (Jiménez-Torres et al., 2012).

Otro de los factores motivacionales que resultan significativos para la población mayor de 50 años es el relacionado con urgencias de salud, lo cual indicaría que se percibe que la práctica de la natación proporcionaría un medio para el manejo efectivo de procesos de salud/enfermedad como lesiones y enfermedades crónicas. Lo anterior coincide con investigaciones en las que se evidencia que los motivos relacionados con el cuidado de la salud resultan primordiales para las personas adultas y adultas mayores (Lee y Oh, 2013; Moreno et al., 2008; Rodríguez-Romo, et al., 2009; Ruiz et al., 2007). A mayor edad las personas tienden a utilizar el ejercicio físico para mejorar sus condiciones de salud 

especialmente al llegar a la vejez (Coto, 2006), por ello, se evidencia un aumento considerable en cuanto al interés por incursionar en actividades físicas por parte de las personas conforme aumenta la edad, con el fin de mejorar aspectos relacionados a la salud (Guedes et al., 2012; Lobo 2009). Estas motivaciones podrían estar asociadas tanto a factores intrínsecos (alcanzar un nivel positivo de salud) como a factores extrínsecos (evitar problemas de salud) (Ingledew et al., 2009).

Por todo lo anterior, se concluye que el sexo y la edad son variables imprescindibles a tomar en consideración en la elaboración de estrategias para fomentar la adherencia al ejercicio físico y la promoción de salud. Además el conocimiento generado en esta investigación posibilita una comprensión y un acercamiento más profundo de la población meta, aspectos que favorecerán para la toma de decisiones futuras en materia de planificación, organización, promoción e intervención en este tipo de proyectos de extensión. Se debe tomar en cuenta que el muestreo por conveniencia y el tamaño de la muestra limitan la posibilidad de extender los hallazgos más allá de la población específica. Estudios posteriores podrían considerar enfoques metodológicos que subsanen esta limitación.

\section{Referencias Bibliográficas}

Águila, C., Sicilia, A., Muyor, J. y Orta, A. (2009). Cultura posmoderna y perfiles de práctica en los centros deportivos municipales. Revista Internacional de Medicina y Ciencias de la Actividad Física y el Deporte, 9(33), 81-95. Recuperado de: http://cdeporte.rediris.es/revista/revista33/artperfiles110.htm

De Andrade, A., Salguero, A. y Márquez, S. (2006). Motivos para a participaçãp esportivaem nadadores brasileiros. Fitness \& Performance Journal, 50(6), 363-369. http://dx.doi.org/10.3900/fpj.5.6.363.p

Benzi, M., \& Polani D. (2000). Motivación y desmotivación. En Tamorri, S. (Ed.), Neurociencias y Deporte (pp. 139-166). Barcelona: Editorial Paidotribo.

Bíró, M., Fügedi, B., \& Revés, L. (2007). The role of teaching swimming in the formation of aconscious healthy lifestyle. International Journal of Aquatic Research and Education, 1(3), 269-284. Recuperado de: http://www.researchgate.net/publication/263946849_The_Role_of_Teaching_Swimmi ng_in_the_Formation_of_a_Conscious_Healthy_Lifestyle_A_Case_Report

Blázquez, A. y Feu, S. (2012). Motivación en actividad física mantenimiento en mujeres de municipios pequeños. Revista Internacional de Medicina y Ciencias de laActividad Física y el Deporte, 12(47), 571-588. Recuperado de: http://cdeporte.rediris.es/revista/revista47/artmotivacion304.htm

Capdevila, L., Niñerola, J. y Pintanel, M. (2004). Motivación y actividad física: El autoinforme de motivos para la práctica de ejercicio físico (AMPEF). Revista de Psicología del Deporte, 13(1), 55-74. Recuperado de: http://www.rpdonline.com/article/view/261/capdevila\%20131 
Cervelló, E., Escartí, A., \& Guzmán, F. (2007). Youth sport dropout from the achievement goal theory. Psicothema, 19(1), 65-71. Recuperado de: http://www.unioviedo.net/reunido/index.php/PST/article/view/8598/8462

Cid, L. y Louro, H. (2010). Praticar natação é uma paixão ou um sacrifício? estudo da relação entre o tipo de paixão que o atleta sente pela modalidade e a sua orientação motivacional. Revista de Iberoamericana de Psicología del Ejercicio y el Deporte, 5(1), 99-114. Recuperado de: http://www.redalyc.org/pdf/3111/311126267008.pdf

Coto, E. (2006). Ejercicio físico y bienestar en personas mayores de 50 años: caso del distrito central del cantón de Turrialba. Inter Sedes, 7(12), 47-59. Recuperado de: http://www.intersedes.ucr.ac.cr/ojs/index.php/intersedes/article/view/139/138

De Andrade, A., Salguero, A., González, R., \& Márquez, S. (2006). Motives for participation in physical activity by Brazilian adults. Perceptual and Motor Skills, 102(2), 358-367. http://dx.doi.org/10.2466/pms.102.2.358-367

Deci, E., \& Ryan, M. (2000). The "what" and "why" of goal pursuits: Human needs and the self determination of behaviour. Psychological Inquiry, 11(4), 227-268. http://dx.doi.org/10.1207/S15327965PLI1104_01

Duran, D., Orbegoz, L. J., Uribe-Rodríguez, A. F. y Uribe, J. M. (2008). Integración social y habilidades funcionales en adultos mayores. Universitas Psychologica, 7(1), 263-270. Recuperado de: http://revistas.javeriana.edu.co/index.php/revPsycho/article/view/213/1691

Gavala, J. (2010). Razones por las que la población de Sevilla realiza práctica físico deportiva. Journal of Sport and Health Research, 3(3), 169-178. Recuperado de: http://www.journalshr.com/papers/Vol\%203_N\%203/V03_3_2.pdf

González, M. T., Landero, R. y Ruiz, M. A. (2008). Modelo estructural predictor de la salud mental y física en mujeres. Revista Panamericana de Salud Pública, 23(2), 101-108. http://dx.doi.org/10.1590/S1020-49892008000200005

Goñi, E. y Infante, G. (2010). Actividad físico-deportiva, autoconcepto físico y satisfacción con la vida. European Journal of Education and Psychology, 3(2), 199-208.

Recuperado de: http://www.ejep.es/index.php/journal/article/view/60/pdf

Granero-Gallegos, A., Gómez-López, M., Abraldes, J.A. y Rodríguez-Suárez, N. (2011). Motivos de práctica en el ámbito de la actividad física no competitiva. Espiral. Cuadernos del Profesorado, 4(7), 15-22. Recuperado de: http://www.abraldes.org/Documentos/00813.pdf

Guedes, D. P., Legnani, R. F. S. y Legnani, E. (2012). Motivos para a práctica de ejercicio físico em universitários e fatores asociados. Revista Brasileira de Educación Física y Esporte, 26(4), 679-89. http://dx.doi.org/10.1590/S1807-55092012000400012 
Guillén, F. y Álvarez-Malé, M. L. (2010). Relación entre los motivos de la práctica deportiva y la ansiedad en jóvenes nadadores de competición. Revista Iberoamericana de Psicología del Ejercicio y el Deporte, 5(2), 233-252. Recuperado de: http://www.gi.ulpgc.es/psicologiadeporte/docs/Relacion-entre-motivos-departicipacion-deportiva-y-ansiedad-en-jovenes-nadadores.pdf

Hritz, N., \& Ramos, W. (2008). To travel or to compete: Motivations of Masters Swimmers. International Journal of Aquatic Research and Education, 2(4), 298-312. Recuperado de:

https://scholarworks.iu.edu/dspace/bitstream/handle/2022/14326/Ramos.pdf?sequence $=1 \&$ isAllowed $=\mathrm{y}$

Ingledew, D., \& Markland, D. (2008). The role of motives in exercise participation.

Psychology and Health, 23(7), 807-828. http://dx.doi.org/10.1080/08870440701405704

Ingledew, D. K., Markland, D., \& Ferguson, E. (2009). Three levels of exercise motivation. Applied Psychology: Health and Well-being, 1(3), 336-355. http://dx.doi.org/j.1758$\underline{0854.2009 .01015 . x}$

Jeffrey, P. (2013). Physical activity behaviors, motivation, and self-efficacy among college students. College Student Journal, 47(1), 64-74. Recuperado de: http://essential.metapress.com/content/15601178j3g37476/

Jepson, R., Harris, F. M., Bowes, A., Robertson, R., Avan, G., \& Sheikh, A. (2012). Physical activity in South Asians: an in-depth qualitative study to explore motivations and facilitators. PLoS One, 7(10), e45333. http://dx.doi.org/10.1371/journal.pone.0045333

Jiménez, M. G., Martínez, P., Miró, E. y Sánchez, A. I. (2008). Bienestar psicológico y hábitos saludables: ¿están asociados a la práctica de ejercicio físico. International Journal of Clinical and Health Phychology, 8(1), 186-202. Recuperado de: http://www.aepc.es/ijchp/articulos_pdf/ijchp-272.pdf

Jiménez-Torres, M. G., Godoy-Izquierdo, D. y Godoy, G. J. F. (2012). Relación entre los motivos para la práctica físico-deportiva y las experiencias de flujo en jóvenes: diferencias en función del sexo. Universitas Psychologica, 11(3), 909-920. Recuperado de: http://www.oalib.com/paper/2380433\#.PDFxahUUXyA

Lee, B. A., \& Oh, D. J. (2013). The effects of health perception on living health belief, living satisfaction and wellbeing-oriented activities according to swimming participation with middle-aged women. Journal of Exercise Rehabilitation, 9(3), 381-388. doi: 10.12965/jer.130047. http://dx.doi.org/10.12965/jer.130047

Lobo, A. (2009). Motivation for physical activity and its relation with the quality of life. Revista Brasileira de Geriatria y Gerontologia, 12(3), 405-415. Recuperado de: http://revista.unati.uerj.br/scielo.php?script=sci_arttext\&pid=S1809$\underline{98232009000300008 \& \operatorname{lng}=\text { pt\&nrm=iso }}$ 
Medic, N., Young, B. W., \& Grove, J. R. (2013). Perceptions of five-year competitive categories: model of how relative age influences competitiveness in masters sport. Journal of Sports Science \& Medicine, 12(4), 724-729. Recuperado de: http://www.jssm.org/vol12/n4/14/v12n4-14pdf.pdf

Mella, R., González, L., D’Appolonio, J., Maldonado, I., Fuenzalida, A. y Díaz, A. (2004). Factores asociados al bienestar subjetivo en el adulto mayor. Psykhe, 13(1), 79-89. http://dx.doi.org/10.4067/S0718-22282004000100007

Moreno, J. y Marín, L. (2002). Diferencias motivacionales en el aprendizaje y desarrollo de programas de natación y de fitness acuático. Fitness \& Performance Journal, 1(2), 4251. http://dx.doi.org/10.3900/fpj.1.2.42.s

Moreno, J., Cervelló, E., y González, D. (2007). Analizando la motivación en el deporte: un estudio a través de la teoría de la autodeterminación. Apuntes de Psicología, 25(1), 3551. Recuperado de: http://www.um.es/univefd/deporteiad.pdf

Moreno, J. A., Cervelló, E., Marcos, P. J. \& Martín, E. H. (2010). Importancia de la valoración del comportamiento autónomo del practicante para el disfrute del practicante en ejercicio físico acuático. Cuadernos de Psicología del Deporte, 10(1), $57-70$.

Moreno, J., Martínez, C., González-Cutre, D. y Marcos, P. (2009). Perfiles Motivacionales de Practicantes en el Medio Acuático frente al Medio Terrestre. Revista Internacional de Medicina y Ciencias de la Actividad Física y del Deporte, 9(34): 201-216. Recuperado de: http://cdeporte.rediris.es/revista/revista34/artperfiles128.pdf

Moreno, J., Cervelló, E. y Martínez, A. (2007). Validación de la Escala de Medida de los Motivos para la Actividad Física-Revisada en españoles: Diferencias por motivos de participación. Anales de psicología, 23(1), 167-176. Recuperado de: http://revistas.um.es/analesps/article/view/23071/22351

Moreno, J., Cervelló, E. y Martínez, A. (2006). Motivación autodeterminada y flujo disposicional en el deporte. Anales de Psicología, 22(2), 310-317. Recuperado de: http://revistas.um.es/analesps/article/view/23291/22571

Moreno-Murcia, J., Borges, F., Marcos, J., Sierra, C., \& Huéscar, E. (2012). Motivación, frecuencia y tipo de actividad en practicantes de ejercicio físico. Revista Internacional de Medicina y Ciencias de la Actividad Física y el Deporte, 12(48), 649-662. Recuperado de: http://cdeporte.rediris.es/revista/revista48/artmotivacion319.pdf

Moreno, J. A., Martínez, C., \& Marcos, P. (2008). Motivations and reasons for exercising in water: Gender and age differences in a sample of Spanish exercisers. International Journal of Aquatic Research and Education, 2, 237-246. Recuperado de: http://www.um.es/univefd/motacua.pdf 
Niñerola, J., Capedvila, L. y Pintanel, M. (2006). Barreras percibidas y actividad física: el autoinforme de barreras para la práctica de ejercicio físico. Revista de Psicología del Deporte, 15(1), 53-69. Recuperado de: http://ddd.uab.cat/pub/revpsidep/19885636v15n1p53.pdf

Pavón, A. y Moreno, J. (2008). Actitud de los universitarios ante la práctica física deportiva: diferencias por géneros. Revista de Psicología del Deporte, 17(1), 723. Recuperado de: http://www.um.es/univefd/acuni.pdf

Pavón, A., Moreno, J., Gutiérrez, M. y Sicilia, A. (2004). Motivos de práctica físico deportiva según la edad y el género en una muestra de universitarios. Ciencias aplicadas a la actividad física y el deporte, 76(2), 13-21. Recuperado de:

http://www.um.es/univefd/motivosuni.pdf

Petri, H. y Govern, J. (2006). Motivación: teoría, investigación y aplicaciones. (pp.16-17). México: Thomson Editores S.A de C.V.

Pérez, V. y Devis, J. (2003). La promoción de la actividad física relacionada con la salud. La perspectiva de proceso y de resultado. Revista Internacional de Medicina y Ciencias de la Actividad Física y el Deporte, 3(10), 69-74. Recuperado de: http://cdeporte.rediris.es/revista/revista10/artpromoci\%f3n.pdf

Pinto, D., Santos, R. y Legani, E. (2012). Motivos para la práctica de ejercicio físico en universitarios de acuerdo con el índice masa corporal. Revista Brasileira de Atividade Física \& Saúde, 17(4), 270-274. Recuperado de: http://www.scielo.br/scielo.php?pid=S198065742013000300009\&script=sci_arttext

Remor, E. (2007). Propuesta de un cuestionario breve para la evaluación de la competitividad en el ámbito deportivo: Competitividad-10. Revista de Psicología del Deporte, 16(2), 167-183. Recuperado de: http://ddd.uab.cat/pub/revpsidep/19885636v16n2p167.pdf

Rodríguez-Romo, G., Boned, C. y Garrido, M. (2009). Motivos y barreras para hacer ejercicio y practicar deportes en Madrid. Revista Panamericana de SaludPública, 26(3), 244254. http://dx.doi.org/10.1590/S1020-49892009000900009

Romero, A. E., Brustad, R. J. y García, A. (2007). Bienestar psicológico y su uso en la psicología del ejercicio, la actividad física y el deporte. Revista Iberoamericana de Psicología del Ejercicio y el Deporte, 2(2), 31-52. Recuperado de: http://www.redalyc.org/pdf/3111/311126258003.pdf

Ruiz, F., García, M. y Díaz, A. (2007). Análisis de las motivaciones de práctica de actividad física y de abandono deportivo en la Ciudad de La Habana (Cuba). Anales de psicología, 23(1), 152-166. Recuperado de: http://revistas.um.es/analesps/article/view/23081/22361 
URL www.una.ac.cr/mhsalud

Rodríguez Montero, A., Morera , M., Barrantes Brais, K., y Ugalde Ramírez, A.

Salguero, A., González-Boto, R., Tuero, C., \& Márquez, S. (2003). Development of a Spanish version of the participation motivation inventory for young competitive swimmers.

Perceptual and Motor Skills, 96(2), 637-646.

http://dx.doi.org/10.2466/pms.2003.96.2.637

Triviño, Z., Stiepovich, J. y Merino, J. M. (2007). Factores predictores de conductas promotoras de salud en mujeres peri-post-menopáusicas de Cali, Colombia. Colombia Medica, 38(4), 395-407. Recuperado de: http://www.scielo.org.co/pdf/cm/v38n4/v38n4a9.pdf

Weiss, M., \& Amorose, A. (2008). Motivational Orientations and Sport Behavior. In Horn, T. (Ed.), Advances in Sport Psycology (pp.116-155). United States: Human Kinetics.

Recepción: 21 de mayo del 2014.

Corrección: 8 de agosto del 2014.

Aceptación: 11 de agosto del 2014.

Publicación: 31 de agosto del 2014. 